Rev. Saúde públ., S. Paulo, 19:458-74, 1985.

\title{
AVALIAÇÃO DO IMPACTO DA SUPLEMENTAÇÃO ALIMENTAR A GESTANTES NO CONTROLE DO BAIXO PESO AO NASCER NO MUNICIPIO DE SÃO PAULO, SP (BRASIL)*
}

\author{
Carlos Augusto Monteiro** \\ Maria Helena D'Aquino Benício** \\ Isildinha Marques dos Reis Lamonica** \\ Solange L'Abbate*** \\ Maria Lúcia Rosa Stefanini*** \\ Julieta Hitomi Oshiro*** \\ Heloisa Bearzotti Pires*** \\ Maria Cecília Goi Porto Alves***
}

\begin{abstract}
MONTEIRo, C.A. et al. Avalação do impacto da suplementação alimentar a gestantes no cotrole do baixo peso ao nascer no município de São Paulo, SP (Brasil). Rev. Saúde públ., S. Paulo, $19: 458-74,1985$.
\end{abstract}

RESUMO: A partir de estudo realizado em oito grandes maternidades do Município de São Paulo, SP (Brasil) que atendem clientela predominantemente de baixo nível sócio-econômico, objetivou-se analisar o impacto da suplementação alimentar durante a assistência pré-natal sobre a incidência de recém-nascidos de baixo peso ao nascer (peso $\leqslant 2.500 \mathrm{~g}$ ). Foram envolvidos no estudo 1.060 recém-nascidos de mães que receberam suplementação e 664 recém-nascidos de mães que não a receberam. A incidência de baixo peso ao nascer foi de cerca de $11 \%$, considerada elevada e semelhante em ambos os grupos de recém-nascidos. A análise multivariada, realizada para controlar eventuais diferenças entre os grupos, que não a condição de suplementação, descartou qualquer associação significativa entre suplementação e peso ao nascer e revelou, por outro lado, que tabagismo e morbidade na gestação e determinadas características antropométricas e reprodutivas da mãe, prévias à gestação, são importantes fatores de risco para o baixo peso ao nascer. A aparente explicação para a ausência de impacto da suplementação alimentar na população estudada parece residir não na quantidade insuficiente da suplementação alimentar oferecida $(370 \mathrm{Kcal} /$ dia), mas no predomínio de fatores não alimentares na determinação do haixo peso ao nascer. São formuladas recomendações quanto ao controle do baixo peso ao nascer no contexto estudado.

UNITERMOS: Gestantes, estado nutricional. Alimentos formulados. Baixo peso ao nascer. Assistência pré-natal.

\section{INTRODUÇÃO}

Considera-se recém-nascido de baixo peso a criança nascida viva que tenha ao nascer peso igual ou inferior a $2.500 \mathrm{~g}$, independentemente da idade gestacional 42 .

Inúmeras são as conseqüências negativas que se associam ao baixo peso ao nascer. Elas envolvem alterações respiratórias e metabólicas de grave repercussão no pós-parto imediato, diminuição da competência imunológica, prejuízo ao crescimento e desenvolvimento pósnatais e sequielas neurológicas manifestas tanto em provas eletroencefalográficas

\footnotetext{
* Pesquisa financiada por Convênio entre a Secretaria de Estado da Saúde do Estado de São Paulo e Ministério da Saúde.

* Do Departamento de Nutrição da Faculdade de Saúde Pública da Universidade de São Paulo Av. Dr. Arnaldo, 715 - 01255 - São Paulo, SP - Brasil.

** Da Secretaria de Saúde do Estado de São Paulo - Av. Dr. Arnaldo, 351 - 01246 - São Paulo, SP - Brasil.
} 
MONTEIRO, C.A. et al. Avaliação do impacto da suplementação alimentar a gestantes no controle do baixo peso ao nascer no Município de São Paulo, SP (Brasil). Rev. Saúde públ.. S. Paulo, 19:458-74, 1985.

quanto em testes de desempenho intelectual 5,10,12,16,39. De resto, estudos sobre mortalidade peri-natal e infantil realizados em vários contextos têm demonstrado que a probabilidade de sobrevivência ao longo do primeiro ano de vida está intimamente relacionada à condição de peso ao nascer ${ }^{6,9,23,2 t}$. Conhecidos diferenciais de mortalidade infantil, como os existentes entre a população americana e a dos países escandinavos ou como aqueles que separam a população não branca da população branca dos Estados Unidos, são atribuídos quase em sua totalidade a diferenças relativas à distribuição de peso ao nascer ${ }^{1,6}$.

A incidência de recém-nascidos de baixo peso é bem conhecida em países desenvolvidos onde o peso ao nascer acompanha o registro de nascimento. Revisões da literatura efetuadas nestes países têm revelado que aquela incidência alcança valores que vão de $5 \%$ em determinados países europeus de altíssimo nível de desenvolvimento, como a Suécia e a Holanda, a 8\% nos Estados Unidos ${ }^{6}$. Resultados que fazem referência a classes sociais revelam desigual distribuição de baixo peso ao nascer, como na Inglaterra 29 e nos Estados Unidos ${ }^{8}$, onde os estratos menos favorecidos da população - Classes IV e $\mathrm{V}$ no primeiro país e população não branca no segundo - apresentam incidências duas vezes superiores às encontradas nos estratos sociais mais favorecidos.

Em países não desenvolvidos, encontram-se apenas estudos esporádicos da distribuição de peso ao nascer, restritos a algumas localidades e, de modo geral, baseados em amostras não representativas. Nestes estudos, têm-se encontrado incidências que variam de 13 a $43 \%{ }^{19}$. A Organização Mundial da Saúde estima que a incidência de recém-nascidos de baixo peso estaria por volta de $7 \%$ nos países desenvolvidos e por volta de
$18 \%$ nos países em desenvolvimento ${ }^{41}$. Em amostra representativa dos recémnascidos em hospitais e maternidades do Município de São Paulo, Monteiro e col. ${ }^{26}$ encontraram incidência de baixo peso ao nascer de $9,7 \%$, aumentando tal incidência para $12,0 \%$, quando consideradas apenas as maternidades públicas.

A epidemiologia do baixo peso ao nascer é complexa, uma vez que muitos fatores parecem exercer influência sobre o peso de nascimento, incluindo características maternas prévias à gravidez, como idade ${ }^{11,37}$, paridade 7,28 e dimensões corporais ${ }^{11,15}$, e características da evolução da gestação, como ganho de peso ${ }^{32,38}$, tabagismo ${ }^{22,25}$, presença de doenças intercorrentes ${ }^{21}$ e recebimento de assistência pré-natal ${ }^{3,30}$.

Um dos fatores mais estudados na determinação do baixo peso ao nascer é o estado nutricional materno durante a gestação. Revendo dados obtidos em experimentos com animais e em estudos epidemiológicos de população, Rosso ${ }^{31}$ analisa minuciosamente a questão e sustenta a tese de que a capacidade de parasitismo do feto é limitada apenas às reservas de nutrientes que a mãe acumula durante a gestação. Segundo aquele autor, quando começam a ser utilizadas reservas maternas pré-concepcionais, desencadear-se-iam alterações nos mecanismos maternos de adaptação à gravidez e como resultado ocorreria um descenso na linha de abastecimento fetal.

Resultados de seis estudos prospectivos de intervenção realizados para avaliar o efeito da suplementação alimentar durante a gestação, concluídos recentemente em diferentes países (Guatemala ${ }^{20}$, México ${ }^{18}$, Colômbia ${ }^{27}$, Formosa ${ }^{40}$, Canadá ${ }^{33}$ e Estados Unidos ${ }^{36}$ ), acrescentaram importantes conhecimentos sobre a inter-relação nutrição materna-nutrição fetal. Nos estudos da Guatemala e México, que abrangeram áreas mui- 
MONTEIRO, C.A. et al. Avaliação do impacto da suplementação alimentar a gestantes no controle do baixo peso ao nascer no Município de São Paulo, SP (Brasil). Rev. Saúde públ., S. Paulo, 19:458-74, 1985.

to pobres destes países, a suplementação determinou significativos incrementos da ordem de $200 \mathrm{~g}$ no peso ao nascer. No estudo da Colômbia e de Formosa observaram-se incrementos no peso ao nascer de cerca de $80 \mathrm{~g}$, os quais não chegaram a ser estatisticamente significativos. No estudo americano e no estudo canadense, realizados respectivamente em Nova York e Montreal, incrementos ligeiros, de cerca de $40 \mathrm{~g}$, foram observados, sendo que em função do número de observações do estudo canadense, o ganho de peso, de pequena magnitude, foi estatisticamente significante.

Revendo em detalhe os estudos prospectivos mencionados, Lechtig ${ }^{17}$ observa que o impacto da suplementação alimentar parece ganhar intensidade à medida que é pior o nível sócio-econômico da população beneficiária e na medida em que é mais deficiente o estado nutricional materno ao início da gestação. A partir desta observação, adverte, com acerto, que o mesmo dramático impacto obtido com a suplementação de gestantes na Guatemala, onde a proporção de baixo peso ao nascer foi reduzida de $33 \%$ para $13 \%$, não deveria ser esperado em populações em melhores condições sócio-econômicas e nutricionais. Lechtig ${ }^{17}$ cita, como exemplo, a população branca americana, cuja incidência de recém-nascidos de baixo peso ao redor de $7 \%$ seria muito pouco afetada pela ação de um programa de suplementação alimentar na gestação.

Em 1977, motivada pelos recém-divulgados e extremamente positivos resultados do estudo de intervenção operado na Guatemala ${ }^{20}$ e preocupada em reduzir a elevada mortalidade infantil $\left(84,35^{\circ} / 00\right.$ ) registrada no Estado (cujo componente neonatal representava metade daquele coeficiente) ${ }^{35}$, a Secretaria da Saúde do Estado de São Paulo implantou, no programa de assistência pré-natal, a atividade de suplementação alimentar a gestantes. Tal implantação foi precedida apenas de um teste de aceitação do suplemento, que foi formulado para ser ingerido entre as principais refeições $e$ fornecer diariamente $o$ equivalente às necessidades calórico-protéicas extraordinárias determinadas pela gravidez.

Apenas em 1980, apresentou-se a oportunidade para se realizar a avaliação da atividade de suplementação iniciada em 1977. Tal avaliação, desenvolvida por pesquisadores da Secretaria de Saúde e da Faculdade de Saúde Pública da Universidade de São Paulo, envolveu a realização de três estudos simultâneos ${ }^{2}$. O primeiro destes estudos buscou avaliar a operacionalização da atividade a nível dos Centros de Saúde do Município de São Paulo. O segundo objetivou avaliar a aceitação e o consumo efetivo do suplemento por parte da clientela daqueles Centros. O terceiro estudo, realizado em oito grandes maternidades do Município de São Paulo, procurou avaliar o impacto que a atividade de suplementação na gestação estaria exercendo sobre a condição de peso ao nascer. Neste artigo serão apresentados e discutidos os resultados obtidos por este último estudo.

\section{METODOLOGIA}

A partir de informações de estudo anterior (Monteiro e col. ${ }^{26}, 1980$ ), realizado em amostra representativa de nascimentos hospitalares ocorridos no Município de São Paulo, foram selecionadas oito maternidades que atendiam predominantemente população de baixo nível sócio-econômico. Seis destas maternidades eram governamentais ou filantrópicas e ofereciam atendimento universal gratuito, da mesma forma que os Centros de Saúde que distribuem a suplementação alimentar na assistência prénatal. As duas outras maternidades eram particulares, porém conveniadas com a Previdência Social e situadas em área 
MONTEIRO, C.A. et al. Avaliação do impacto da suplementação alimentar a gestantes no controle do baixo peso ao nascer no Município de São Paulo, SP (Brasil). Rev. Saúde públ., S. Paulo, 19:458-74, 1985 .

geográfica altamente favorável à captação de clientela previdenciária de baixo nível sócio-econômico.

Em 1980, em cada uma das maternidades selecionadas, durante período de 55 dias, foram entrevistadas todas as puérperas que haviam dado a luz no dia anterior ao da entrevista. Foram, assim, identificados três grupos de gestantes: as que haviam recebido assistência pré-natal e suplementação alimentar na gestação (cerca de $20 \%$ do total das entrevistadas), as que haviam recebido apenas assistência pré-natal (cerca de 50\%) e as que não haviam recebido nenhuma assistência na gestação (cerca de $30 \%$ ). Para este estudo, foram selecionadas todas as gestantes do primeiro grupo (1.060 gestantes, doravante denominadas grupo com suplementação) e uma amostra das gestantes do segundo grupo (664 gestantes, doravante denominadas grupo sem suplementação). Uma amostra do grupo de gestantes sem assistência pré-natal foi selecionada, mas não será abordada neste estudo.

Para todas as gestantes do grupo suplementado, a suplementação recebida referiu-se ao produto Gestal, mistura em pó que é distribuída nos serviços estatais de pré-natal localizados no Município de São Paulo. Cada $100 \mathrm{~g}$ da referida mistura fornece $370 \mathrm{Kcal}$ e $10 \mathrm{~g}$ de proteína e, como rotina, são entregues a cada gestante $3 \mathrm{~kg}$ da mistura por mês, com a orientação de que o produto seja dissolvido em água ou em outro veículo e consumido nos intervalos entre as refeições. A estimativa para a quantidade média de suplemento recebida por gestante (avaliada a partir de $98 \%$ dos casos para os quais a informação era suficientemente precisa) foi de $9.870 \mathrm{~g}$ ( $\pm 2.127 \mathrm{~g})$.

A estimativa para a quantidade efetivamente consumida por gestante, ou seja, a quantidade recebida subtraída da quantidade do produto eventualmente não consumido ou fornecido a outrém (avaliada a partir de $90 \%$ dos casos para os quais a informação era suficientemente precisa) foi de $6.798 \mathrm{~g}( \pm 2.107 \mathrm{~g})$.

Para o controle "a posteriori" de eventuais diferenças entre os grupos, que não a condição de suplementação, e também para o estudo do efeito da suplementação em gestações submetidas a diferentes condições predisponentes de baixo peso ao nascer, as puérperas pertencentes aos dois grupos foram entrevistadas, obtendo-se das mesmas, além de informações referentes ao recebimento e consumo de suplemento, dados sócio-econômicos, características reprodutivas, perfil antropométrico antes da gravidez e história de tabagismo e de intercorrências capazes de comprometer o crescimento fetal.

A partir das informações obtidas, as gestações de ambos os grupos foram classificadas conforme estivessem ou não submetidas a condições predisponentes de baixo peso, reconhecidas pela literatura.

Em uma primeira etapa da análise, o possível efeito da suplementação sobre o peso ao nascer foi estudado a partir da proporção de recém-nascidos de baixo peso, encontrada nos grupos com e sem suplementação. Foram efetuadas comparações tanto para o conjunto dos recémnascidos dos dois grupos quanto para sub-grupos de recém-nascidos submetidos a diferentes condições predisponentes de baixo peso. Estas últimas comparações visaram surpreender uma eventual diferenciação do efeito da suplementação conforme a presença de riscos e, também, exercer um primeiro controle sobre eventuais diferenças quanto à constituição dos grupos com e sem suplementação.

Em uma segunda etapa da análise, os dados referentes ao conjunto dos nascimentos estudados foram submetidos à análise multivariada pela técnica de modelos log-lineares na forma logito ${ }^{4,34}$, 
MONTEIRO, C.A. et al. Avaliação do impacto da suplementação alimentar a gestantes no controle do baixo peso ao nascer no Município de São Paulo, SP (Brasil). Rev. Saúde públ., S. Paulo, 19:458-74, 1985.

fixando-se a condição de peso ao nascer como variável resposta (baixo peso não baixo peso) e empregando-se a presença da suplementação e a presença de condições predisponentes de baixo peso como variáveis independentes. Como se verá, a técnica de análise multivariada adotada permite identificar e quantificar a associação que a presença da suplementação mantém com o peso ao nascer, controlados os efeitos das demais variáveis estudadas. Também possibilita comparar o efeito exercido pela suplementação com os efeitos que poderiam ser esperados com outras intervenções (como o controle do tabagismo, por exemplo). Finalmente, a técnica utilizada permite estudar eventuais modificações do efeito da suplementação em sub-grupos de gestantes expostas a condições predisponentes de baixo peso ao nascer.

\section{RESULTADOS}

Caracteristicas Gerais e Presença de Fatores Predisponentes a Baixo Peso ao Nascer nos Dois Grupos

A Tabela 1 descreve as características gerais dos grupos de puérperas com e sem suplementação alimentar durante a gestação.

Como era de se esperar, em função das características das maternidades onde se desenvolveu o trabalho, em ambos os grupos de puérperas predominam condições próprias de população de baixo nível sócio-econômico: renda insuficiente, baixa escolaridade, dimensões corporais reduzidas e excessiva morbidade. São também condições frequientes nos dois grupos a existência de mães sem companheiro, a paridade nos extremos do período reprodutivo e o consumo de cigarros na gestação. Por outro lado, em ambos os grupos, mais de $90 \%$ das puérperas receberam três ou mais consultas durante a assistência pré-natal.
Comparados entre si, os grupos com e sem suplementação apresentam diferenças de pequena magnitude, sendo a maioria delas destituída de significação estatística. Em relação a características sócio-econômicas, a comparação é desvantajosa para o grupo suplementado, sendo significativas as diferenças quanto à renda e proporção de mães analfabetas. Em relação a características reprodutivas, pouco diferem os dois grupos, havendo apenas um predomínio ligeiro e não significativo de paridades antes dos 18 e partir dos 35 anos no grupo suplementado. Em relação a dimensões corporais, são significativamente menores no grupo suplementado as médias de peso e de adequação do peso à altura, embora, mais uma vez, sejam de pequena magnitude as diferenças observadas. Quanto às características de gestação que foram levantadas - morbidade, tabagismo e assistência pré-natal - os dois grupos praticamente nada diferem.

Objetivando comparar os dois grupos de modo mais direto quanto à presença de fatores comumente associados a maior incidência de recém-nascidos de baixo peso, construiu-se a Tabela 2, onde são apresentadas as proporções dos nascimentos dos dois grupos que estiveram submetidos, alternativamente, às seguintes condições predisponentes de baixo peso ao nascer:

- presença de um ou mais "fatores de risco" decorrentes de características sócio-econômicas adversas ("renda per capita < 0,30 salários mínimos", "analfabetismo materno", "mãe sem companheiro");

- presença de um ou mais "fatores de risco" decorrentes de características reprodutivas adversas ("idade materna $<$ 18 ou $\geqslant 35$ anos", "paridade 4 ou +", "intervalo interpartal $<1$ ano"); 
MONTEIRO, C.A. et al. Avaliação do impacto da suplementação alimentar a gestantes no controle do baixo peso ao nascer no Município de São Paulo, SP (Brasil). Rev. Saúde públ., S. Paulo, 19:458-74, 1985.

\section{TABELA 1}

Características gerais dos grupos com e sem suplementação alimentar durante a gestação (São Paulo, 1980)

\begin{tabular}{|c|c|c|c|c|c|}
\hline Características & $\begin{array}{r}\text { su } \\
\mathrm{N} \\
\end{array}$ & $\begin{array}{l}\text { Frupo com } \\
\text { plementação } \\
\text { alimentar }\end{array}$ & & $\begin{array}{l}\text { Grupo sem } \\
\text { uplementação } \\
\text { alimentar }\end{array}$ & $\begin{array}{c}\text { Significado estatístico } \\
\text { da diferença } \\
\text { entre os grupos }\end{array}$ \\
\hline \multicolumn{6}{|l|}{$\begin{array}{l}\text { Características } \\
\text { sócio-econômicas }\end{array}$} \\
\hline $\begin{array}{l}\text { Renda per capita } \\
\text { (em salários mínimos) }\end{array}$ & 960 & & 621 & & \\
\hline $\begin{array}{l}\overline{\mathrm{x}} \pm \mathrm{dp} \\
\%<0,50 \\
\%<0,30\end{array}$ & & $\begin{array}{c}0,89 \pm 0,65 \\
31,4 \\
11,4\end{array}$ & & $\begin{array}{c}1,14 \pm 0,94 \\
21,7 \\
8.1\end{array}$ & $\begin{array}{l}\mathrm{p}<.001 \\
\mathrm{p}<.001 \\
\mathrm{p}<.05\end{array}$ \\
\hline $\begin{array}{l}\text { N. }{ }^{\circ} \text { de pessoas na } \\
\text { família }\end{array}$ & 1.060 & & 664 & & \\
\hline$\overline{\mathrm{X}} \pm \mathrm{dp}$ & & $4,0 \pm 2,3$ & & $4,0 \pm 2,3$ & NS \\
\hline $\begin{array}{l}\text { Escolaridade do chefe } \\
\text { da família }\end{array}$ & 975 & & 629 & & \\
\hline$\%$ analfabetos & & 16,5 & & 13,2 & NS \\
\hline $\begin{array}{l}\text { \% c/ primário } \\
\text { completo ou mais }\end{array}$ & & 52,7 & & 42,5 & NS \\
\hline Escolaridade da mãe & 1.054 & & 661 & & \\
\hline$\%$ analfabetas & & 16,3 & & 11,2 & $\mathrm{p}<.01$ \\
\hline $\begin{array}{l}\text { \% c/ primário } \\
\text { completo ou mais }\end{array}$ & & 44,7 & & 48,4 & NS \\
\hline Relação marital & 1.060 & & 664 & & \\
\hline $\begin{array}{l}\% \text { mães sem } \\
\text { companheiro }\end{array}$ & & 22,4 & & 18,8 & NS \\
\hline \multicolumn{6}{|l|}{$\begin{array}{l}\text { Características } \\
\text { reprodutivas }\end{array}$} \\
\hline $\begin{array}{l}\text { Idade materna } \\
\text { (em anos) }\end{array}$ & 1.060 & & 664 & & \\
\hline$\overline{\mathrm{X}} \pm \mathrm{dp}$ & & $24,6 \pm 6,1$ & & $25,2 \pm 5,8$ & NS \\
\hline$\%<18$ & & 7,7 & & 5,4 & NS \\
\hline$\% \geq 35$ & & 8,4 & & 7,9 & NS \\
\hline Paridade & 1.060 & & 664 & & \\
\hline $\bar{X} \pm d p$ & & $2,8 \pm 2,1$ & & $2,8 \pm 2,3$ & NS \\
\hline$\% 1$ & & 40,0 & & 37,5 & NS \\
\hline$\% 4 \mathrm{e}+$ & & 26,9 & & 24,4 & NS \\
\hline $\begin{array}{l}\text { Intervalo interpartal } \\
\text { (dias) para os não } \\
\text { primogênitos }\end{array}$ & 636 & & 415 & & \\
\hline$\overline{\mathrm{X}} \pm \mathrm{dp}$ & & $1.134,7 \pm 776,6$ & & $1.145,5 \pm 851,0$ & NS \\
\hline$\%<365$ & & 4,7 & & 5,3 & NS \\
\hline
\end{tabular}


MONTEIRO, C.A. et al. Avaliação do impacto da suplementação alimentar a gestantes no controle do baixo peso ao nascer no Município de São Paulo, SP (Brasil). Rev. Saúde públ.. S Paulo, 19:458-74, 1985.

(Continuação da Tabela 1)

\begin{tabular}{|c|c|c|c|c|c|}
\hline Características & $\mathrm{N}$ & $\begin{array}{l}\text { upo com } \\
\text { imentação }\end{array}$ & & $\begin{array}{l}\text { upo sem } \\
\text { mentação }\end{array}$ & $\begin{array}{c}\text { Significado estatístico } \\
\text { da diferença } \\
\text { entre os grupos }\end{array}$ \\
\hline \multicolumn{6}{|l|}{$\begin{array}{l}\text { Características } \\
\text { antropométricas da } \\
\text { mãe }\end{array}$} \\
\hline $\begin{array}{l}\text { Peso prévio à } \\
\text { gravidez }(\mathrm{kg})\end{array}$ & 996 & & 629 & & \\
\hline $\bar{X} \pm d p$ & & $52,9 \pm 8,1$ & & $54,4 \pm 9,1$ & $\mathrm{p}<.01$ \\
\hline$\%<45$ & & 11.7 & & 10,5 & NS \\
\hline Altura $(\mathrm{cm})$ & 1.053 & & 660 & & \\
\hline $\bar{X} \pm d p$ & & $157,3 \pm 6,6$ & & $157,7 \pm 6,6$ & NS \\
\hline$\%<150$ & & 10,4 & & 9,5 & NS \\
\hline $\begin{array}{l}\text { Adequação do peso } \\
\text { à altura }(\%)\end{array}$ & 990 & & 626 & & \\
\hline $\bar{X} \pm d p$ & & $96,7 \pm 13,5$ & & $99,2 \pm 15,0$ & $\mathrm{p}<.01$ \\
\hline$\%<80$ & & 7,5 & & 6.9 & NS \\
\hline \multicolumn{6}{|l|}{$\begin{array}{l}\text { Características da } \\
\text { gestação }\end{array}$} \\
\hline $\begin{array}{l}\text { \% com intercorrên- } \\
\text { cias próprias da } \\
\text { gravidez }\end{array}$ & & 8,9 & & 9.9 & NS \\
\hline $\begin{array}{l}\text { \% com intercorrên- } \\
\text { cias infecciosas } \\
\text { sistêmicas e/ou } \\
\text { vulvo-vaginais }\end{array}$ & & 10,7 & & 11,4 & Yis \\
\hline $\begin{array}{l}\text { \% com outras inter- } \\
\text { corrências com } \\
\text { repercussão po- } \\
\text { tencial para a } \\
\text { nutrição fetal }\end{array}$ & & 3,3 & & 2,6 & NS \\
\hline $\begin{array}{l}\text { \% com combinações } \\
\text { de intercorrências }\end{array}$ & & 4,4 & & 2.6 & NS \\
\hline Tabagismo & 1.059 & & 663 & & \\
\hline$\%$ fumantes & & 40.6 & & 39,0 & NS \\
\hline $\begin{array}{l}\% 6 \text { cigarros ou } \\
\text { mais / dia }\end{array}$ & & 21,0 & & 23,4 & NS \\
\hline $\begin{array}{l}\text { N. }{ }^{\circ} \text { médio cigar- } \\
\text { ros/dia nas fumantes }\end{array}$ & & $9,1 \pm 8,0$ & & $10,2 \pm 7,7$ & NS \\
\hline Assistência pré-natal & 1.060 & & 664 & & \\
\hline$\% 3$ consultas e mais & & 92,2 & & 90,7 & NS \\
\hline
\end{tabular}


MONTEIRO, C.A. et al. Avaliação do impacto da suplementação alimentar a gestantes no controle do baixo peso ao nascer no Município de São Paulo, SP (Brasil). Rev. Saúde públ., S. Paulo, 19:458-74, 1985.

- presença de um ou mais "fatores de risco" decorrentes de características antropométricas adversas prévias à gravidez ("peso $<45 \mathrm{~kg}$ ", "altura $<150$ cm", "adequação do peso à altura < $80 \% ") *$;

- presença de intercorrência na gravidez capaz de potencialmente prejudicar a nutrição fetal **;

- presença de consumo de 6 ou mais cigarros.

A observação dos resultados da $\mathrm{Ta}$ bela 2 revela, inicialmente, que, em to- das as condições predisponentes de baixo peso ao nascer consideradas pelo estudo, tanto o grupo suplementado quanto o não suplementado apresentam elevada frequiência de nascimentos submetidos a risco. $\mathrm{Na}$ comparação entre os grupos, verifica-se que, com exceção do tabagismo, em todas as outras condições de risco o grupo suplementado apresenta maior frequêencia de nascimentos atingidos, embora as diferenças sejam relativamente pequenas e significativas apenas para a presença de fatores de risco formulados a partir das características sócio-econômicas dos grupos.

\section{TABELA 2}

Proporções de nascimentos submetidos a risco de baixo peso ao nascer nos grupos com e sem suplementação conforme diferentes características maternas consideradas na determinação do risco (São Paulo, 1980)

\begin{tabular}{|c|c|c|c|c|c|}
\hline \multirow{2}{*}{$\begin{array}{l}\text { Características } \\
\text { maternas } \\
\text { consideradas }\end{array}$} & \multicolumn{2}{|c|}{$\begin{array}{c}\text { Grupo com } \\
\text { suplementação }\end{array}$} & \multicolumn{2}{|c|}{$\begin{array}{c}\text { Grupo sem } \\
\text { suplementação }\end{array}$} & \multirow{2}{*}{$\begin{array}{l}\text { Significado estatístico } \\
\text { da diferença entre } \\
\text { os grupos }\end{array}$} \\
\hline & $\begin{array}{l}\text { N. }{ }^{\circ} \text { de } \\
\text { nascim. } \\
\text { observ. }\end{array}$ & $\begin{array}{c}\% \text { sob } \\
\text { risco }\end{array}$ & $\begin{array}{l}\mathrm{N} \cdot{ }^{\circ} \mathrm{de} \\
\text { nascim. } \\
\text { observ. }\end{array}$ & $\begin{array}{c}\% \text { sob } \\
\text { risco }\end{array}$ & \\
\hline Sócio-econômicas & 954 & 38,4 & 618 & 29,6 & $\mathrm{p}<.001$ \\
\hline Reprodutivas & 1.019 & 36,9 & 652 & 32,7 & NS \\
\hline Antropométricas & 990 & 20,5 & 626 & 18,8 & NS \\
\hline $\begin{array}{l}\text { Morbidade na } \\
\text { gestação }\end{array}$ & 1.060 & 27,4 & 664 & 25,0 & NS \\
\hline $\begin{array}{l}\text { Tabagismo na } \\
\text { gestação }\end{array}$ & 1.059 & 21,0 & 663 & 23,4 & NS \\
\hline
\end{tabular}

* Ou seja, peso inferior a $80 \%$ do peso médio esperado para a altura segundo os padrões antropométricos de referência sugeridos por Julliffe 13 .

** Foram considerados, neste caso, os três grupos de intercorrências referidos na Tabela 1, quais sejam, intercorrências próprias da gravidez (síndromes hipertensivas, placenta prévia etc.), intercorrências de oriegm infecciosa, sistêmicas ou vulvo-vaginais (infecção urinária, gastroenterites, cervicites etc. e intercorrências outras cuja descrição autorizava supor prejuízos ao crescimento fetal (hiperemese persistente, anemia severa e outras). 
MONTEIRO, C.A. et al. Avaliação do impacto da suplementação alimentar a gestantes no controle do baixo peso ao nascer no Município de São Paulo, SP (Brasil). Rev. Saúde públ., S. Paulo, 19:458-74, 1985.

Peso ao Nascer nos Grupos Com e Sem Suplementação

A Tabela 3 apresenta as estatísticas referentes ao peso ao nascer dos dois grupos estudados. Confirmando a vulnerabilidade a que estão submetidos os nascimentos de ambos os grupos, os resultados obtidos apontam médias de peso ao nascer aquém das esperadas em populações normais e incidência relativamente alta de recém-nascidos de baixo peso. Na forma em que estão apresentados os resultados, isto é, sem levar em conta as diferenças existentes entre os grupos quanto a fatores capazes de influenciar o peso ao nascer, não há diferenças significativas entre os mesmos.

TABELA 3

Peso ao nascer nos grupos com e sem suplementação alimentar durante a gestação (São Paulo, 1980)

\begin{tabular}{lccc}
\hline Peso ao nascer (g) & $\begin{array}{c}\text { Grupo com } \\
\text { suplementação } \\
\mathrm{n}=1.060\end{array}$ & $\begin{array}{c}\text { Grupo sem } \\
\text { suplementação } \\
\mathrm{n}=664\end{array}$ & $\begin{array}{c}\text { Significado estatístico } \\
\text { das diferenças entre } \\
\text { os grupos }\end{array}$ \\
\hline$\% \leqslant 2.500$ & 10,9 & 11,0 & NS \\
$\overline{\mathrm{X}} \pm \mathrm{dp}$ & $3.087 \pm 523$ & $3.123 \pm 560$ & $\mathrm{NS}$ \\
\hline
\end{tabular}

$\mathrm{Na}$ tentativa de exercer algum controle sobre as diferenças existentes entre os grupos e ao mesmo tempo averiguar a influência da suplementação conforme a presença ou ausência de determinadas condições predisponentes de baixo peso ao nascer, construiu-se a Tabela 4 . Ali pode-se verificar que na presença ou au- sência de cinco diferentes condições de risco para o baixo peso (as mesmas explicitadas na Tabela 2), continuam não se.ldo estatisticamente significativas as diferenças entre os grupos suplementado e não suplementado. Duas constatações adicionais fluem da Tabela 4 e merecem ser destacadas.

\section{TABELA 4}

Proporção (\%) de recém-nascidos de baixo peso nos grupos com e sem suplementação conforme presença ou ausência de risco induzido por diferentes características maternas (São Paulo, 1980)

\begin{tabular}{llccc}
\hline Características maternas & $\begin{array}{c}\text { Condição } \\
\text { de risco }\end{array}$ & $\begin{array}{c}\text { Grupo com } \\
\text { suplementação }\end{array}$ & $\begin{array}{c}\text { Grupo sem } \\
\text { suplementação }\end{array}$ & $\begin{array}{c}\text { Significado } \\
\text { estatístico das } \\
\text { diferenças entre os } \\
\text { grupos }\end{array}$ \\
\hline \multirow{2}{*}{ Sócio-econômicas } & Presente & 11,7 & 11,5 & NS \\
& Ausente & 10,4 & 10,6 & NS \\
Reprodutivas & Presente & 12,2 & 15,0 & NS \\
Antropométricas & Ausente & 9,6 & 9,1 & NS \\
Morbidade & Presente & 14,3 & 17,8 & NS \\
& Ausente & 10,0 & 8,9 & NS \\
Tabagismo & Presente & 14,5 & 14,5 & NS \\
& Ausente & 9,6 & 9,8 & NS \\
& Presente & 15,3 & 20,6 & NS \\
\hline
\end{tabular}


MONTEIRO, C.A. et al. Avaliação do impacto da suplementação alimentar a gestantes no controle do baixo peso ao nascer no Município de São Paulo, SP (Brasil). Rev. Saúde públ., S. Paulo, 19:458-74, 1985.

- Conforme esperado, em ambos os grupos, a presença de qualquer uma das cinco condições de risco implica elevar a incidência de baixo peso ao nascer, ainda que no caso específico da presença de características sócio-econômicas desfavoráveis (baixa renda, analfabetismo materno e ausência de companheiro), tenha sido mínima aquela elevação.

- Apenas em três situações pôde-se registrar tendência de o grupo suplementado apresentar menor incidência de baixo peso ao nascer: na presença de tabagismo, na presença de condiçōes antropométricas maternas desfavoráveis e na presença de características reprodutivas adversas.

\section{Estudo Controlado do Efeito da Suplementação}

Buscando-se o controle simultâneo de todas as cinco condições predisponentes de baixo peso consideradas pelo estudo, realizou-se a análise multivariada dos dados relativos aos casos para os quais todas as informações eram disponíveis (866 casos no grupo suplementado e 576 no grupo não suplementado). Conforme já mencionado, a técnica utilizada foi a de modelos log-lineares na forma de logito, a qual permite conhecer a parcela de ação de diferentes variáveis independentes (neste caso, suplementação e condições predisponentes de baixo peso) sobre uma dada variável resposta (neste caso, peso ao nascer), além de permitir identificar eventuais efeitos combinados de variáveis independentes sobre a variável resposta.

A análise multivariada começou com a obtenção de um modelo inicial, denominado saturado, em que comparecem parâmetros relativos a todas as possíveis associações entre variáveis independentes e entre estas e a variável resposta. A seguir, em operações sucessivas, pela exclusão de parâmetros não significativos, foram sendo obtidos modelos gradativamente mais simples, até se chegar ao modelo escolhido.

No modelo saturado, obtido inicialmente, verificou-se não haver significado estatístico em nenhum dos parâmetros relativos às associações de mais de uma variável independente com a variável resposta. Nesta medida, invalidou-se a possibilidade de existirem efeitos combinados significativos sobre o peso ao nascer, descartando-se com isto a hipótese de que a suplementação pudesse exercer efeitos diferenciados sobre o peso ao nascer conforme a condição de risco da gestante.

A exclusão dos parâmetros relativos às associações entre mais de uma variável independente com a variável resposta determinou que o primeiro modelo alternativo ao modelo saturado incluísse apenas associações de primeira ordem com a variável resposta, no caso as associações que suplementação e condições predisponentes de baixo peso mantêm individualmente com peso ao nascer.

O Anexo 1 detalha as etapas sucessivas de simplificação a que foi submetido o primeiro modelo alternativo encontrado (Modelo 1), iniciadas com a tentativa de retirada de parâmetros não significativos (Modelos 2, 3, 4 e 5) e culminadas com a tentativa de retirada de parâmetros significativos (Modelos 6, 7,8 e 9). A partir dali, verificou-se que o Modelo 5, que suprimiu os parâmetros relativos às associações entre suplementação e peso ao nascer e condições sócioeconômicas e peso ao nascer, apresentou o mesmo excelente ajuste $(\mathrm{p}=0,97)$ obtido pelo Modelo 1, denotando com isto não serem suplementação e condições sócio-econômicas fatores definidores da condição de peso ao nascer (o que, aliás, já se antevia na análise não multivariada). 
MONTEIRO, C.A. et al. Avaliação do impacto da suplementação alimentar a gestantes no controle do baixo peso ao nascer no Município de São Paulo, SP (Brasil). Rev. Saúde públ., S. Paulo, 19:458-74, 1985.

Ainda no mesmo Anexo 1, verifica-se que as tentativas posteriores de simplificação do Modelo 5 não foram bem sucedidas, pois implicaram perda significativa de ajuste. Tal fato determinou que o Modelo 5 fosse o modelo final escolhido pela análise multivariada. A partir deste modelo, pôde-se concluir que, dentre as variáveis estudadas, apenas as condições reprodutivas e antropométricas da mãe e a morbidade e o tabagismo durante a gestação exerceram influência significativa sobre o peso ao nascer.

Na Tabela 5, apresentam-se os parâmetros do modelo escolhido referentes às associações significativas com $o$ peso ao nascer.

$\mathrm{Na}$ Tabela 6 são apresentadas as frequiências ajustadas pelo modelo para o peso ao nascer na ausência das quatro condições predisponentes de baixo peso e na presença individual de cada uma delas. A mesma tabela apresenta a razão dos produtos cruzados ("odds ratio") que caracteriza a presença exclusiva de cada uma das quatro condições enfocadas (em estimativa pontual e por intervalo). Apresenta, também, a incidência de recém-nascidos de baixo peso esperada na ausência e na presença de cada uma daquelas condições.

TABELA 5

Estimativas dos parâmetros do modelo escolhido referentes às associações entre peso ao nascer e diferentes condições de risco

\begin{tabular}{lccc}
\hline Condição de risco & $\begin{array}{c}\text { Estimativa } \\
\text { do parâmetro }\end{array}$ & Desvio padrão & $\begin{array}{c}\text { Estimativa } \\
\text { padronizada }\end{array}$ \\
\hline Reprodutiva & 0,086 & 0,053 & 1,620 \\
Antropométrica & 0,155 & 0,053 & 2,906 \\
Morbidade & 0,151 & 0,053 & 2,838 \\
Tabagismo & 0,188 & 0,053 & 3,528 \\
\hline
\end{tabular}

TABELA 6

Frequiências ajustadas de peso ao nascer, razão dos produtos cruzados ("odds ratio") e incidência de baixo peso ao nascer conforme a presença de determinadas condições de risco (São Paulo, 1980)

\begin{tabular}{lccccc}
\hline \multirow{2}{*}{$\begin{array}{l}\text { Presença de condições } \\
\text { de risco }\end{array}$} & $\begin{array}{c}\text { Frequências ajustadas } \\
\text { Peso ao nascer }\end{array}$ & $\begin{array}{c}\text { Baixo } \\
\text { peso }\end{array}$ & $\begin{array}{c}\text { Não baixo } \\
\text { peso }\end{array}$ & $\begin{array}{c}\text { Razão dos produtos } \\
\text { cruzados * }\end{array}$ & $\begin{array}{c}\text { Incidência do baixo } \\
\text { peso ao nascer }\end{array}$ \\
\cline { 2 - 5 } Nenhuma & 11,14 & 160,86 & & & $6,5 \%$ \\
Morbidade & 6,19 & 48,81 & 1,83 & {$[1,21-2,77]$} & $11,2 \%$ \\
T.ibagismo & 7,05 & 47,95 & 2,12 & {$[1,40-3,21]$} & $12,8 \%$ \\
A:Itropométrica & 5,59 & 43,41 & 1,86 & {$[1,23-2,82]$} & $11,4 \%$ \\
Reprodutiva & 7,40 & 75,60 & 1,41 & {$[0,93-2,14]$} & $8,9 \%$ \\
\hline
\end{tabular}

* Sendo $A$ e $B$ as frequiências ajustadas de baixo peso e não baixo peso na ausência de fatores de risco e $C$ e $D$ as mesmas freqüências ajustadas para a presença de um determinado fator, a razão dos produtos cruzados referentes àquele fator é dada por $\mathrm{B} \times \mathrm{C} / \mathrm{A} \times \mathrm{D}$. 
MONTEIRO, C.A. et al. Avaliação do impacto $d_{\omega}$ suplementação alimentar a gestantes no controle do baixo peso ao nascer no Município de São Paulo. SP (Brasil). Rev. Saúde públ., S. Paulo, 19:458-74, 1985.

Os resultados da Tabela 6 revelam que a presença de tabagismo na gestação (mais de cinco cigarros diários, conforme definido) representa o maior risco de baixo peso, implicando em mais do que dobrar a incidência daquela condição ("odds ratio" de 2,12).

A seguir surgem as condições antropométricas maternas (peso prévio à gestação $<45 \mathrm{~kg} \mathrm{e} /$ ou altura $<150 \mathrm{~cm}$ e/ou peso/altura $<80 \%$, conforme definido) e intercorrências patológicas na gestação capazes de quase dobrar a incidência de baixo peso ao nascer ("odds ratio" de 1,86 e 1,83 , respectivamente). Finalmente, encontram-se as condições reprodutivas maternas (idade $<18$ anos $\mathrm{e} /$ ou paridade $4 \mathrm{e}+\mathrm{e} / \mathrm{ou}$ intervalo interpartal < de 1 ano, conforme definido) capazes de aumentar apenas ligeiramente a incidência de baixo peso ao nascer ("odds ratio" de 1,41).

Uma vez que o modelo é multiplicativo ${ }^{3}$, a estimativa de risco de baixo peso devido à presença simultânea de mais de uma condição desfavorável é obtida pelo produto dos riscos referentes a cada uma delas. Assim, a presença simultânea de todas as condições aventadas na Tabela 6 aumentaria em mais de dez vezes a incidêneia de baixo peso correspondente à ausência de risco.

\section{DISCUSSÃO E CONCLUSÕES}

O achado principal da avaliação conduzida é o de que a atividade de entrega de suplementação alimentar durante a assistência pré-natal não foi capaz de reduzir a excessiva incidência de recémnascidos de baixo peso observada entre gestantes clientes da rede oficial de Centros de Saúde do Município de São Paulo.

Considerando que cuidados metodológicos apropriados foram tomados - dimensão da amostra, consideração de grupo-controle, controle de variáveis, verificação de efeitos combinados, entre outros - passa a ser importante examinar razões que expliquem $o$ insucesso registrado e que possam orientar o planejamento de outras formas de intervenção.

Três razões, desde logo, surgem como possíveis explicações:

1 - A quota de suplemento oferecida mensalmente às gestantes seria insuficiente para fazer frente ao déficit alimentar de sua dieta habitual.

2 - Embora a quota oferecida fosse teoricamente adequada às necessidades, a quantidade de suplemento efetivamente consumida pela gestante não chegaria a ser suficiente.

3 - O déficit alimentar não seria o principal determinante de baixo peso ao nascer na população estudada, predominando nesta determinação fatores não decorrentes da alimentação e, portanto, não passíveis de correção por suplementação alimentar.

O exame conclusivo das três razões referidas dependeria de informações não inteiramente conhecidas no estudo e disponíveis, possivelmente, apenas em investigações de tipo prospectivo. As considerações feitas a seguir permitem apenas apreciar a procedência das mesmas.

Durante a assistência pré-natal conduzida nos Centros de Saúde são colocados à disposição da gestante $3 \mathrm{~kg}$ de suplemento por mês. Tanto as gestantes suplementadas quanto as não suplementadas iniciaram a assistência pré-natal, em mais de $90 \%$ das vezes, no primeiro ou no segundo trimestre, tendo os dois grupos permanecido no programa em média cerca de 5 meses. Teoricamente, portanto, a quantidade média total de suplemento colocado à disposição das gestantes suplementadas estaria em torno de $15 \mathrm{~kg}$. Tal quantidade corresponde a $55.500 \mathrm{Kcal}$ e $1.500 \mathrm{~g}$ de proteína, o que se aproxima tanto dos custos calóricoprotéicos extraordinários referentes à gra- 
MONTEIRO, C.A. et al. Avaliação do impacto da suplementação alimentar a gestantes no controle do baixo peso ao nascer no Município de São Paulo, SP (Brasil). Rev. Saúde públ., S. Paulo, 19:458-74, 1985.

videz ${ }^{14}$ quanto do consumo médio de suplemento registrado em intervenções de suplementação bem sucedidas como as operadas na Guatemala ${ }^{20}$ e México ${ }^{18}$.

Não parece procedente, assim, atribuir à quantidade de suplemento oferecido a ausência de impacto registrada pela avaliação.

A partir das entrevistas efetuadas com as puérperas nas maternidades, pôde-se averiguar que nem sempre as gestantes suplementadas levaram sua quota integral mensal de suplemento, na maioria das vezes tal fato devendo-se a estoques presentes no domicílio e formados em face do não consumo regular do produto oferecido ("sobras"). Em virtude desta ocorrência, a quantidade média de suplemento efetivamente recebida pelas gestantes, conforme mencionado no capítulo de metodologia, reduziu-se a cerca de $10 \mathrm{~kg}$. Conforme também já mencionado, pôde-se apurar que parte do suplemento recebido foi consumido por outras pessoas do domicílio ou mesmo dado a vizinhos e parentes, o que implica, finalmente, reduzir-se a cerca de $7 \mathrm{~kg}$ a quantidade média de fato consumida pelas gestantes.

A redução da quantidade consumida a menos da metade da quantidade teoricamente planejada, ainda que não explique inteiramente a ausência de efeito da suplementação, determina que sejam naturalmente menores as expectativas quanto a seu impacto.

A terceira razão cogitada para explicar o insucesso da suplementação em promover a redução da incidência de baixo peso ao nascer refere-se à possibilidade de, na população estudada, não serem déficits alimentares os fatores predominantes na determinação do baixo peso. Ainda que, como nas vezes anteriores, seja difícil ser conclusivo quanto à procedência da razão aventada, três ordens de observações indubitavelmente falam a seu favor:
1) A frequiente formação de estoques de suplemento no domicílio ("sobras") e a doação do produto a vizinhos e parentes, denotando ser improvável um déficit calórico acentuado na alimentação materna.

2) A magnitude da incidência do baixo peso ao nascer na população estudada, cerca de $11 \%$, que está longe de se igualar às incidências duas a três vezes superiores observadas quando déficits alimentares são de grande magnitude e praticamente universais como nas localidades investigadas da Guatemala e México, onde, como já se referiu, a suplementação alimentar se mostrou bastante eficaz.

3) A freqüente ocorrência na população estudada de inquestionáveis fatores de risco de baixo peso ao nascer não decorrentes da alimentação da gestante, como tabagismo, morbidade na gestação e baixa estatura materna.

As três observações, em conjunto, tornam plausível a hipótese de que a ausência de impacto da suplementação alimentar tenha sido sobretudo decorrência do predomínio de fator's não alimentares na determinação do baixo peso ao nascer.

Embora reconhecendo a natureza complexa da determinação do baixo peso ao nascer e a necessidade de investigações adicionais quanto a seu controle a nível de Saúde Pública, os achados da avaliação efetuada permitem que, desde já, algumas recomendações sejam feitas aos responsáveis pela assistência pré-natal no Município de São Paulo:

- não parece haver sentido em se manter a atividade de entrega de suplementação alimentar na assistência prénatal de forma indiscriminada como vem se fazendo; tal entrega deveria ser reservada a casos individuais em que a avaliação do estado nutricional da gestante 
MONTEIRO, C.A. et al. Avaliação do impacto da suplementação alimentar a gestantes no :ontrole do baixo peso ao nascer no Município de São Paulo, SP (Brasil). Rev. Saúde públ., S. Paulo, 19:458-74, 1985.

(não efetuada presentemente de forma sistemática na assistência pré-natal) assim o indicasse;

- se estudos ulteriores confirmarem a aparente tendência de alguns grupos especiais de gestantes se beneficiarem da suplementação, como por exemplo as submetidas a condições antropométricas desfavoráveis ao início da gravidez (Tabela 3), poderia ser indicado estender às mesmas a entrega de suplemento;

- dada a elevada frequiência na população estudada de fatores de risco não alimentares, como o tabagismo e a morbidade na gestação, e dada a vulnerabilidade potencial destes fatores a ações factíveis ao nível da assistência pré-natal convencional, seria imperativo rever o conteúdo programático da assistência que vem sendo prestada às gestantes, de mo- do a adequá-lo eficazmente ao perf 1 l de problemas encontrados.

Finalmente, uma observação adicional, que decorre dos resultados obtidos com a avaliação efetuada, é a de que o simples registro de incidência excessiva de recém-nascidos de baixo peso, mes. mo que acompanhado de indicadores populacionais sugestivos de baixo nível sócio-econômico, não garante que sejam meramente alimentares os determinantes daquele problema e, menos ainda, que se possa esperar da suplementação alimentar a solução para seu controle. Neste caso, como em muitos outros, verifica-se que as intervenções operadas pela Saúde Pública nem sempre são precedidas do apropriado diagnóstico do problema a ser enfrentado e do criterioso exame de alternativas disponíveis.

MONTEIRO, C.A. et al. [An assessment of the impact of supplementary feeding to pregnant women on low birth weight rates in $S$. Paulo city (Brazil)]. Rev. Saúde públ., S. Paulo, $19: 458-74,1985$

ABSTRACT: The impact of supplementary feeding to pregnant wornen on the incidence of low birth weight $(\leqslant 2500 \mathrm{~g})$ was investigated in 8 maternity hospitals which attend low socioeconomic population in the city of S. Paulo, Brazil. Only mothers who had received prenatal care were studied: 1060 supplemented during pregnancy and 664 unsupplemented during pregnancy. The incidence of low birth weight was similarly high in both groups: around $11 \%$. A multivariate analysis done for controlling possible confounding variables failed to demonstrate any association between supplementation and birth weight. On the other hand, the same multivariate analysis demonstrated that smoking and disease during pregnancy and certain maternal anthropometric and reprodutive characteristics previous to pregnancy are important high risk factors for low birth weight. The most likely explanation for the absence of impact of maternal supplementation on birth weight does not appear to lie in the quantity of food offered to mothers $(370 \mathrm{Kcal} /$ day), but on the relative predominance of non dietary factors in the etiology of low birth weight in the population studied. Recommendations for the control of low birth weight are made with this in mind.

UNITERMS: Pregnancy, nutritional status. Food, formulated. Infant, low birth weight. Prenatal care. 
MONTEIRO, C.A. et al. Avaliação do impacto da suplementação alimentar a gestantes no controle do baixo peso ao nascer no Município de São Paulo, SP (Brasil). Rev. Saúde públ., S. Paulo, 19:458-74, 1985 .

\section{REFERENCIAS BIBLIOGRÁFICAS}

1. ARMSTRONG, R.J. A study of infant mortality from linked records by birth weight, period of gestation, and other variables. United States, 1960 live-birth cohort. Vital Hith Statist. Ser. 20. (12) May, 1972.

2. AVALIAÇÃO da atividade de suplementação alimentar do Programa de Assistência à Gestante da Secretaria de Estado da Saúde de São Paulo: metodologia. São Paulo, Secretaria de Estado da Saúde/Faculdade de Saúde Pública da Universidade de São Paulo, 1982. v. 1 .

3. BENICIO, M.H.D'A. et al. Análise multivariada de fatores de risco para o baixo peso ao nascer em nascidos vivos do município de São Paulo. Rev. Saúde públ., S. Paulo, 19:311-20, 1985

4. BISHOP, Y.M.M. et al. Discrete multivariate analysis: theory and practice. Cambridge, Mass., The MIT Press, 1980.

5. CHANDRA, R.K. Fetal malnutrition and postnatal immunocompetence. Amer. $J$. Dis. Child., 129:450-4, 1975.

6. CHASE, H.C. International comparison of perinatal and infant mortality: the United States and six West European Countries. Vital Hlth Statist. Ser. 3, (6) Mar. 1967.

7. CHASE, H.C. Time trends in low birth weight in the Unites States, 1950-1974. In: Reed, D.M. \& Stanley, F., eds. Epidemiology of prematurity. Baltimore, Urban \& Schwarzenberg, 1977. p. 17-34.

8. CHASE, H.C. \& BYRNES, M.E. Trends in "prematurity": United States, 1950-67. Vital Hlth Statist. Ser. 3, (15) Jan. 1972.

9. ERHARDT, C.L. et al. Influence of weight and gestational age on perinatal and neonatal mortality by ethnic group. Amer. J. publ. Hlth, 54:1841-55, 1964.

10. FITZHARDINGE, P.M. \& STEVEN, E.M. The small-for-date infant. II Neurological and intellectual sequelae. Pediatrics, 50:50-7, 1972

11. HARDY, J.B. \& MELLITS, E.D. Relationship of low birth weight to maternal characteristics of age, parity, education and body size. In: Reed, D.M. \& Stanley. F., ed. Epidemiology of prema- turity. Baltimore, Urban \& Schwarzenberg, 1977. p. 105-16.

12. HARPER, P.A. \& WIENER, G. Sequelas of low birth weight. Ann. Rev. Med. 16:405-20, 1965 .

13. JELLIFFE, D.B. Evaluación del estado de nutrición de la comunidad. Ginebra, Organizacion Mundial de la Salud, 1968. (OMS - Serie de monografias, 53).

14. JOINT FAO/WHO AD HOC EXPERT COMMITTEE ON ENERGY AND PROTEIN REQUIREMENTS, Rome, 1971. Report. Geneva, World. Health Organization, 1973. WHO-Tecn. Rep. Ser., 522).

15. KAMINSKI, M. et. al. Prediction of low birth weight and prematurity by a multiple regression analysis with maternal characteristics known since the beginning of the pregnancy. Int. J. Epidem., 2:195-204, 1973.

16. LASKY, R.E. et al. Birth weight and psychomotor performance in rural Guatemala. Amer. J. Dis. Child., 129:566. 70,1975

17. LECHTIG, A. Early malnutrition, growth and development. Brasília, 1983. [Mimeografado].

18. LECHTIG, A., ed. Effects of maternal nutrition on infant health: implications for action; An international workshop, Guatemala, 1979. Panayachel, Guatemala, 1979.

19. LECHTIG, A. et al. Maternal nutrition and fetal growth in developing societies: socieconomic factors. Amer. J. Dis. Child., 129:434-7, 1975.

20. LECHTIG, A. et al. Effect of food supplementation during pregnancy on birthweight. Pediatrics, 56:508-20, 1975.

21. LECHTIG, A. et al. Effect of morbidity during pregnancy on birth weight in a rural Guatemalan population. Ecol. Food Nutr., 5:225-33, 1976.

22. LOWE, C.R. Effect of mothers' smoking habits on birth weight of their children. Brit. med. J., 10:673-6, 1959.

23. MATA, L. The children of Santa Maria Cauqué: a prospective study of health and growth. Cambridge, Mass., The MIT Press, 1977 
MONTEIRO, C.A. et al. Avaliação do impacto da suplementação alimentar a gestantes no controle do baixo peso ao nascer no Município de São Paulo, SP (Brasil). Rev. Saúde públ., S. Paulo, 19:458-74, 1985 .

24. MCKEOWN, T. \& GIBSON, J.R. Observations on all births. $(23,790)$ in Birmingham, 1947. IV - "Premature birth". Brit. med. J., 3:513-7, 1951.

25. MEYER, M.B. Effects of material smoking and altitude on birth weight and gestation. In: Reed, D.M. \& Stanley F.J., eds. The epidemiology of premaiurity. Baltimore, Urban \& Schwarzenberg, 1977. p. 81-104.

26. MONTEIRO, C.A. et al. A distribuição do peso ao nascer no município de São Paulo, Brasil. Rev. Saúde públ., S. Paulo, 14:161-72, 1980.

27. MORA, J.Q. et al. Nutritional supplementation and the ouctome of pregnancy. I. Birth weight. Amer. J. clin. Nutr. 32:455-62, 1979.

28. PRATT, M.W. et al. National variations in prematurity (1973 and 1974). In: Reed, D.M. \& Stanley, F., eds. Epidemiology of prematurity. Baltimore, Urban \& Schwarzenberg, 1977. p. 53-79.

29. PETROS-BARVAZIAN, A. \& BEHAR, M. Socio economic factors and birth weight distribution. In: Stersky, G. \& Mellander, L. Birth weight distribution: an indicator of social development; $\mathrm{Re}$ port from a SAREC/WHO Workshop. Uppsala, Swedish Agency for Research Cooperation with Developing Countries, 1978. p. $39-41$

30. QUICK, J.D. et al. Prenatal care and pregnancy outcome in an HMO and general population: a multivariate cohort analysis. Amer. J. publ. Hlth, 71:381-90, 1981.

31. ROSSO, P. Nutrición y intercambio materno-fetal: una perspectiva diferente. Arch. lat. amer. Nutr., 27 (supl. 1): 13446, 1977.

32. ROSSO, P. Nutrition and maternal-fetal exchange. Amer. J. clin. Nutr., 34:74455, 1981.

33. RUSH, D. Nutritional services during pregnancy and birth-weight: a retrospective matched pair analysis. Canad. med. Ass. J., 125:567-76, 1981.
34. SOUZA, J.M.P. de \& BENICIO, M.H.D'A. Análise multivariada: um exemplo usando modelo log-linear. Rev. Saúde públ., S. Paulo, 19:263-9, 1985.

35. SUPLEMENTAÇÃO alimentar a gestantes para reduzir coeficiente de mortalidade infantil. Saúde, S. Paulo, 1(2): 1, nov. 1976

36. SUSSER, M. Prenatal nutrition, birthweight and psychological development: an overview of experiments, quasi-experiments and natural experiments in the past decade. Amer. J. clin. Nutr., 34: 784-803, 1981.

37. TAFFEL, S. Factors associated with low birth weight. Vital Hlth Statist. Ser. 21 (37) Apr. 1980.

38. VAN DEN BERG, B.J. Maternal variables affecting fetal growth. Amer. J. clin. Nutr., 34:722-6, 1981.

39. WIENER, G.R. et al. Correlates of low birth weight: psychological status at eight to ten years of age. Pediat. Res., 2:110-8, 1968 .

40. WOHLLEB, J.C, et al. The Bacon Chow Study: maternal supplementation and infant growth. Early hum. Develop., 9: 79-81, 1983.

41. WORLD HEALTH ORGANIZATION. Division of Family Health. The incidence of low birth weight: a critical review of available information. Wld Hlth Statist. Quart., 33: 197-204, 1980

42. WORLD HEALTH ORGANIZATION. Expert Committee on the Prevention of Perinatal Mortality and Morbidity, Geneva, 1969. Report. Geneva, 1970. (Techn. Rep. Ser., 457).

Recebido para publicação em 21/03/1985 Aprovado para publicação em 08/07/1985 
MONTEIRO, C.A. et al. Avaliação do impacto da suplementação alimentar a gestantes no controle do baixo peso ao nascer no Município de São Paulo, SP (Brasil). Rev. Saúde públ., S. Paulo, 19:458-74, 1985.

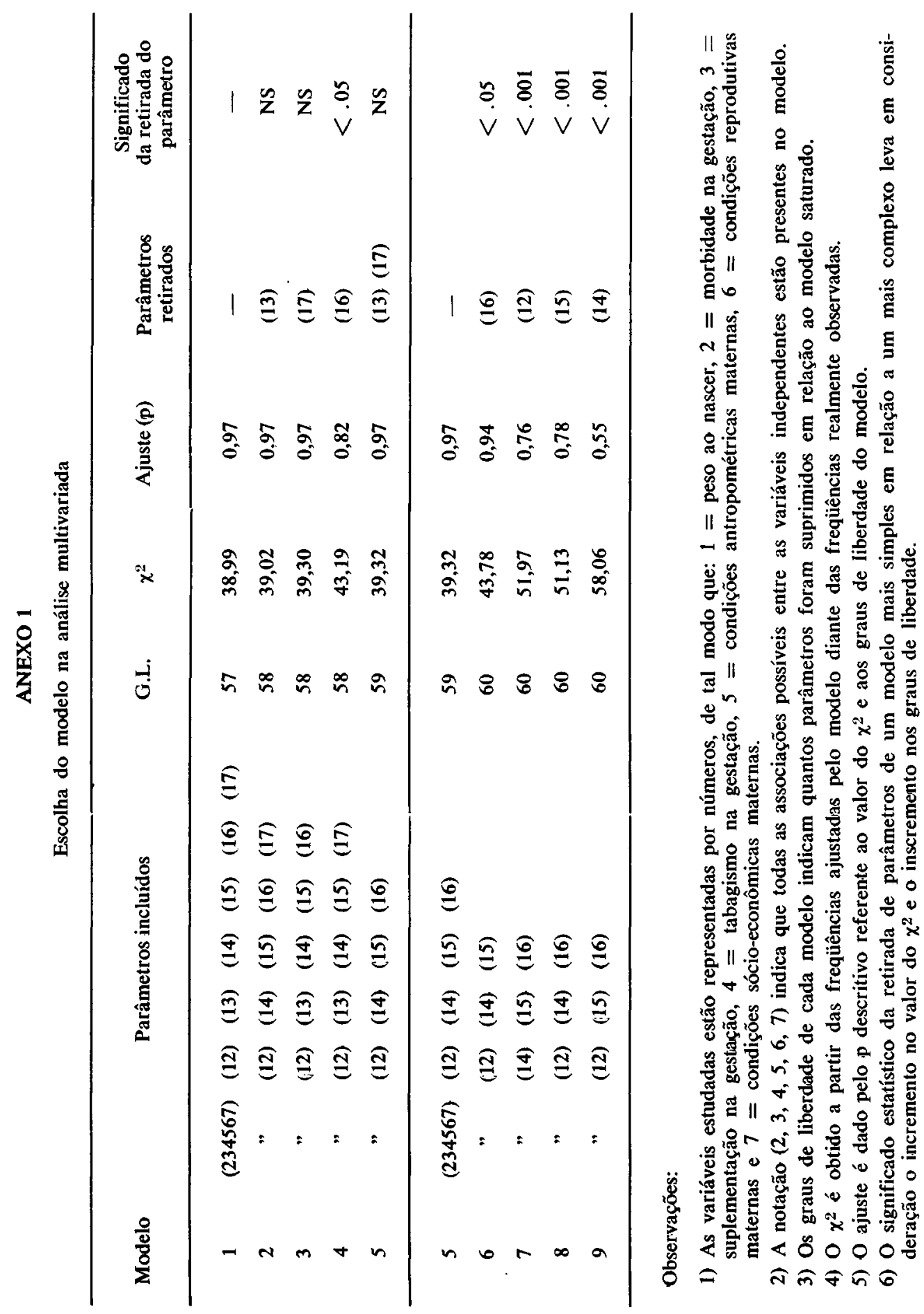

